\title{
Control and analysis software for a laser scanning microdensitometer
}

\author{
H R BUNDEL, C P NAVATHE, P A NAIK and P D GUPTA \\ Laser Plasma Division, Raja Ramanna Centre for Advanced Technology, Indore \\ 452013, India \\ e-mail: bundel@cat.ernet.in
}

MS received 25 July 2005; revised 29 December 2005

\begin{abstract}
A PC-based control software and data acquisition system is developed for an existing commercial microdensitometer (Biomed make model No. SL2D/1D UV/VIS) to facilitate scanning and analysis of X-ray films. The software is developed in Labview, which includes operation of the microdensitometer in 1D and 2D scans and analysis of spatial or spectral data on X-ray films, such as optical density, intensity and wavelength. It provides a user-friendly Graphical User Interface (GUI) to analyse the scanned data and also store the analysed data/image in popular formats like data in Excel and images in jpeg. It has also on-line calibration facility with standard optical density tablets. The control software and data acquisition system is simple, inexpensive and versatile.
\end{abstract}

Keywords. Microdensitometer; optical density; Labview.

\section{Introduction}

Scanning microdensitometers (Moghe et al 1990; Vora et al 2002) are widely used in many scientific research laboratories for optical density (OD) measurements of X-ray/visible light spectra and characterization of the radiation source images (such as laser beams, X-ray emission from plasma etc.) recorded on photographic films. Although detectors like CCD camera facilitate on-line recording and processing of the data, films are also used in many situations due to their availability in large sizes, high dynamic range, and high resolution. Normally for line spectrum analysis, films are scanned by microdensitometer in one-dimensional (1D) mode. However, radiation source images are characterized by scanning the film in twodimensional mode. 2D scanning is not only important to analyse X-ray images of hot plasma, but the same densitometer is used for scanning medical films (for tests like ELISA etc.) where 2D scanning is essential. We have developed a PC-based control and data acquisition system for operating an existing commercial microdensitometer (Biomed model No. SL-2D/1D UV/VIS) with 1D/2D scan and on-line calibration facility. This paper presents important features of this control system and the software for the same. 


\section{Description of the system}

Figure 1 shows a schematic diagram of the microdensitometer and the data acquisition system. Light from the source (He-Ne laser) is focused on the film to be scanned through a pinhole to improve the beam quality of the laser. Light transmitted through the film falls on a photocell and output of the photocell is given to a voltage amplifier.

The film to be scanned is placed on a glass slide and its movement is controlled by a translational stage driven by a stepper motor. It can scan in both $X$ and $Y$ directions up to film length of $100 \mathrm{~mm}$ with a scanning speed of $\sim 2 \mathrm{~mm} / \mathrm{s}$. The photocell output signal depends on the intensity of transmitted light falling on the photocell through the film. The amplified output of the photocell is used as analog input for the data acquisition system. Mechanical limit switch has been used for the detection of initial position of the slide.

\section{Interfacing with PC}

As shown in figure 1, the interfacing of microdensitometer is done using a multi-function general purpose data acquisition card (NI model No. PCI6023E) which is mounted in a PCI

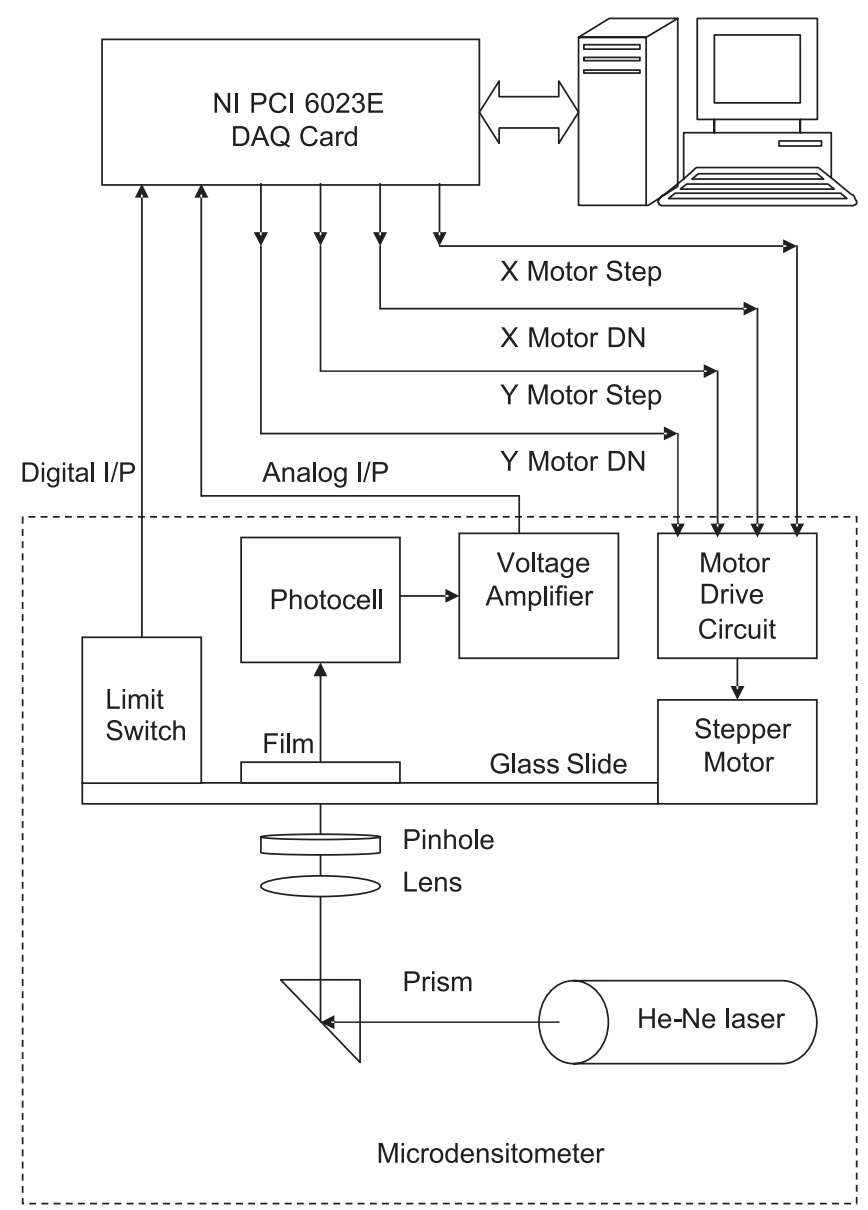

Figure 1. Schematic block diagram of the microdensitometer and the control and data acquisition system. 
slot of a Pentium-4 based industrial computer. This card has 16 single-ended analog input channels, a 12-bit analog to digital converter (ADC) with a sampling rate of $200 \mathrm{k}$ samples per second, 32 digital input/output lines and two general purpose 24-bit timer (counter). One timer has been configured to generate a pulse train of $200 \mathrm{~Hz}$ with a TTL-compatible amplitude for driving the $X$ stepper motor, while the other one is similarly configured for $Y$ stepper motor of the translational stage. Two digital lines are used for setting direction of motion of the stepper motors and one line is used for sensing initial start position of the film to be scanned. The analog signal from the photocell is given as input to the ADC and its digital output is used for recording the transmitted signal as well as analysis of the recorded spectra on the films. This complete program is user-friendly and developed using Labview.

\section{Programming requirement and implementation}

Data acquisition system for this application was designed to carry out following functions:

1. Scan the X-ray films in $1 \mathrm{D}$ or $2 \mathrm{D}$ mode,

2. store the same in digital form,

3. provide a user-friendly Graphical User Interface (GUI) for analysis, and

4. store the analyzed data/image in popular formats like data in Excel and images in jpeg.

This software consists of different modules, such as 'Reset', '1D Scan', '2D Scan', and 'Analysis' programs, which are developed in Labview, a general purpose programming software used to develop Windows-based programs. It is a graphical programming language unlike traditional texts based programming languages. It is a very user-friendly programming language with very strong library functions and it also supports direct input/output programming.

\section{Scanning program}

Scanning program consists of two different programs for $1 \mathrm{D}$ and $2 \mathrm{D}$ scans. This is because in 1D scan mode, a film is scanned in $X$ direction only, but in 2D scan mode, it is scanned in both $X$ and $Y$ directions. In 1D scan mode, for $100 \mathrm{~mm}$ scan length with scanning speed of $\sim 2 \mathrm{~mm} / \mathrm{s}$ and maximum resolution of $\sim 10$ micron, the time required for a single scan is $\sim 1$ minute (including returning time). But in 2D scan mode, it would take about 167 hours with same resolution and also data file size would also become very large. Therefore we have optimized the $Y$ direction scanning resolution up to $\sim 100$ micron maximum in 2D scan mode to bring down the scanning time up to practical limits $\sim 17$ hours for full length scan. However, the 2D spectra images normally scanned have a size of $\sim 25 \mathrm{~mm}$, so that the scanning time and data file size are not unduly large.

\subsection{1 scan program}

This mode is developed for scanning a film in one direction only. In this mode, initially the user has to run the 'Reset' program to bring the translational stage to its initial position. The film is then mounted on the glass slider and the ' $1 \mathrm{D}$ scan' program is run. The user is prompted to enter essential variable parameters such as scan length and input signal range. When a command is given to start scanning, the motor moves one step in the forward direction, the 
ADC is triggered to take one sample of the signal from the photocell and the digitized signal of that element is stored in the memory. In this way, the program scans the film up to the length set by the user and then stops. Due to "move one step, take one sample, and then take the next step" sequence, the spatial resolution remains unaffected by change of speed. Then the program displays a plot of transmission versus scanned film length using scanned data of the film and also saves the same in a user-defined text file.

\subsection{2 scan program}

In this mode also, the user has to initially run the 'Reset' program to bring the translational stage to its initial position. After mounting the film on the glass slider, the user has to start ' $2 \mathrm{D}$ scan' program, which first moves the film in forward direction up to the full length set by the user and then comes back to its initial $X$ position. Then the $Y$ stepper motor moves one step in the forward $Y$ direction, followed again by the $X$ motor scanning in forward direction. In this way, the film is scanned up to the preset $X$ and $Y$ lengths by the user. The ADC records the signal from the photocell in each step. When scanning is over, the program displays an intensity graph of scanned data versus $X Y$ position and also saves the same in a user-defined format.

It may be noted that it is necessary to scan a standard OD tablet (in 1D) before scanning the film in order to get calibrated readout of $\mathrm{OD}$, for both $1 \mathrm{D}$ and 2D scans.

\section{Analysis program}

After scanning the film in 1D or 2D mode, the 'Analysis' program can be started by the user to analyse the scanned data. It asks for previously stored scanned data file name and displays scanned data versus position plots in different formats, which are described in the following section.

\subsection{D analysis mode}

In this mode, 1D scanned data files are analysed in the following formats as required by the user:

6.1a Transmission versus position: In this analysis, the photocell signal versus position of the film data is plotted using calibration of the measured OD with standard OD tablets. For this purpose, the user is required to scan the standard OD tablet in a reference file. This program initially displays the data as transmission versus scanned position plot. The user then reads the measured transmission ( $T_{\text {measured }}=\mathrm{ADC}$ signal/signal with no film) values from the plot of scanned reference OD tablet and provides corresponding standard OD values for the same. The OD values provided by the user are converted into corresponding transmission using the relation $T_{\text {calculated }}=10^{-O D}$. Then the program linearly fits the relationship in measured and calculated transmission values, and calculates the slope $(m)$ and intercepts $(c)$ of the fitted line. The calculated slope and intercept are applied directly in the measured transmission of the films, using the following formula,

$$
T_{\text {Calibrated }}=m \times T_{\text {measured }}+c .
$$

Thus calibrated transmission for the whole scan is calculated and plotted as transmission versus position. 
6.1b Compliment of transmission (ADC scale data) vs position: In this analysis, the photocell signal is subtracted from the full scale signal (i.e. signal when the film is absent) of the ADC. Since the scanning is done in transmission mode, the higher the OD of the spectral line, the lower the transmitted signal. For better visibility of spectral lines recorded on the film, the measured signal is subtracted from the full scale value of the ADC and displayed. This mode is useful to have a quick look at the spectrum without going into detailed analysis.

6.1c OD versus position: This is calculated by converting calibrated transmission data into optical density using the standard relation,

$$
O D_{\text {calibrated }}=\log _{10}\left(1 / T_{\text {calibrated }}\right) \text {. }
$$

Then these data are plotted against the positions.

6.1d $O D$ vs wavelength: In this analysis, initially the user marks the wavelengths of two or three spectral lines in the recorded spectrum with the help of the standard densitogram and the corresponding scanned spatial position, and enters the same in a table provided by the software. The program then calculates relationship between the scanned position and the wavelength, by calculating constants up to third order as desired by the user.

$$
\lambda=a_{0}+a_{1} x+a_{2} x^{2}+a_{3} x^{3},
$$

where, $a_{0} a_{1} a_{2} a_{3}$ are constants and $x$ is the measured position.

The calculated constants are then applied in the above equation to convert spatial (scanned position) data into wavelength data.

6.1e Intensity versus wavelength: In this analysis, the previously calculated OD is converted to intensity to determine intensity of the spectral lines using the following relation,

$$
\text { Intensity }=10^{(O D / \gamma)},
$$

where $\gamma$ is a film constant to be provided by the user.

\section{$6.22 \mathrm{D}$ analysis mode}

This mode is used to analyse the 2D scanned data files. As in 1D analysis, the user runs the $2 \mathrm{D}$ analysis program and provides the name and location of the scanned data file to be analysed. After taking the data from the scanned data file, the program displays a 3D plot, using $X, Y$ positions and the corresponding digitalized data. In 2D mode also, the measured transmission data can be converted to OD or intensity as required by the user. Figure 2 shows a typical $2 \mathrm{D}$ analysed data image. This image is actually an X-ray line spectrum of silicon plasma recorded using a crystal based X-ray spectrograph, recorded on a Kodak DEF-5 Xray film. The lines in the picture are X-ray emission lines from hydrogen-like silicon ions $\left(\mathrm{Si}^{+13}\right)$ in plasma produced by focusing a $5 \mathrm{~J}, 5 \mathrm{~ns}$ Nd:glass laser pulse on a solid silicon target, focused to an intensity of $5 \times 10^{12} \mathrm{~W} \mathrm{~cm}^{-2}$. The three prominent spectral lines are seen as "mountain ridges" in the 3D plot. The software also has a facility to plot the $X$ position (or wavelength) versus data (i.e. transmission or OD or intensity) and also $Y$ position versus (i.e. transmission or OD or intensity) as described for 1D analysis. The analysed data can also be saved for further analysis in other Windows-based software programmes. The 2D analysed data images can also be saved in jpeg format for further processing or use. 


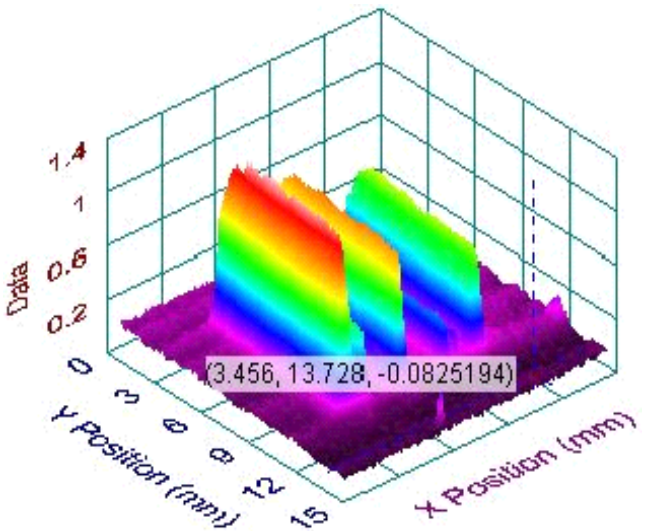

Figure 2. 3D plot of an image of the X-ray spectral lines from silicon plasma recorded on a film.

\section{Results}

The operation and performance of the microdensitometer with on line-calibration is tested by scanning and plotting of the spatial variation of optical densities for the various X-ray films exposed by laser produced plasma. Although we have used the instrument with X-ray films only, it must be noted that the same can be done for visible light spectrum/image etc. recorded on standard black and white films. Optical density up to 2.5 with a resolution of $0.01 \mathrm{OD}$ is measured by scanning various X-ray films. It may be noted here that one can reach an OD of 2.5 with this instrument only because it uses an $\mathrm{He}-\mathrm{Ne}$ laser as the source of light. Other sources, like for example, a laser diode used as a source in standard image scanners, does not have sufficient intensity to reach such ODs. A spatial resolution of $\sim 70$ micron is measured by scanning different size of the standard pin holes. As mentioned earlier, in our "move-stop-measure-move" sequence, the spatial resolution becomes independent of the scanning speed. Figure 3 shows a scan plot of the raw data obtained by the data acquisition system from a magnesium X-ray spectrum of magnesium plasma produced by using a single-nanosecond laser ( $7 \mathrm{~J}$ in $5 \mathrm{~ns}$ ) pulse focused to an intensity of $\sim 7 \times 10^{12} \mathrm{~W} \mathrm{~cm}^{-2}$. The spectrum is recorded on Kodak DEF-5 X-ray film. Figure 4 shows the corresponding final processed data plot of wavelength versus intensity, in which raw data are converted into intensity by using the appropriate $\gamma$ value $(0 \cdot 8)$ of the film and the distance is converted to wavelength. Various prominent lines in the spectral range of $6.5 \AA$ to $9.6 \AA$ are identified as transitions in He-like $\left(\mathrm{Mg}^{+10}\right)$ and H-like $\left(\mathrm{Mg}^{+11}\right)$ magnesium ions, viz. $\mathrm{Mg}(\mathrm{XI}) 1 s^{2}-1 s 5 p$ at $\lambda=7 \cdot 31 \AA$, $\mathrm{Mg}(\mathrm{XI}) 1 s^{2}-1 s 4 p$ at $\lambda=7.47 \AA$,

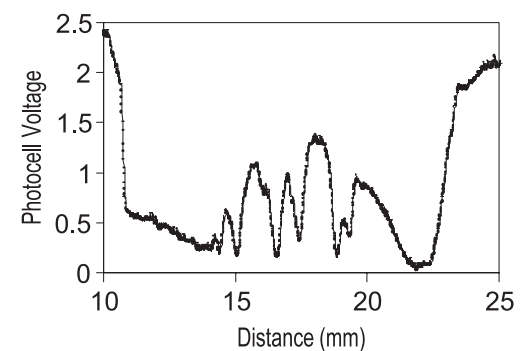

Figure 3. Photocell voltage versus distance (raw data) plot of an X-ray spectrum. 


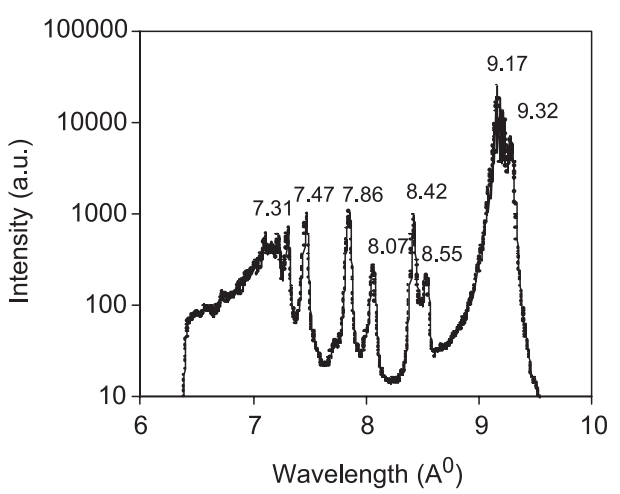

Figure 4. Intensity versus wavelength plot of the data in figure 3 after full 1D analysis.

$\mathrm{Mg}(\mathrm{XI}) 1 s^{2}-1 s 3 p$ at $\lambda=7 \cdot 86 \AA$, 2 s satellite of $\mathrm{Mg}(\mathrm{XI}) 1 s^{2}-1 s 3 p$ at $\lambda=8 \cdot 07 \AA, \mathrm{Mg}$ (XII) $1 s-2 p$ at $\lambda=8.42 \AA, S, J$ satellites of $\mathrm{Mg}$ (XII) $1 s-2 p$ at $\lambda=8.55 \AA, \mathrm{Mg}(\mathrm{XI})$ $1 s^{2}-1 s 2 p$ at $\lambda=9.17 \AA, \mathrm{Mg}(\mathrm{XI}) 1 s^{2}-1 s 2 p$ at $\lambda=9.23 \AA$, and $j, k$ satellites of $\mathrm{Mg}$ (XI) $1 s^{2}-1 s 2 p$ at $\lambda=9 \cdot 32 \AA$. This trace also matches very well with the standard spectral dispersion. The spectral resolution depends upon the spectrograph used to record the signal and microdensitometer used to scan the film. However the spatial resolution of the film (grain size) or source size can limit the spectral resolution. In the present case, the spectral resolution is limited by the X-ray source size. The spectral resolution in this spectrum is unaffected by the microdensitometer spectral resolution because the spectral resolution of the spectrum is

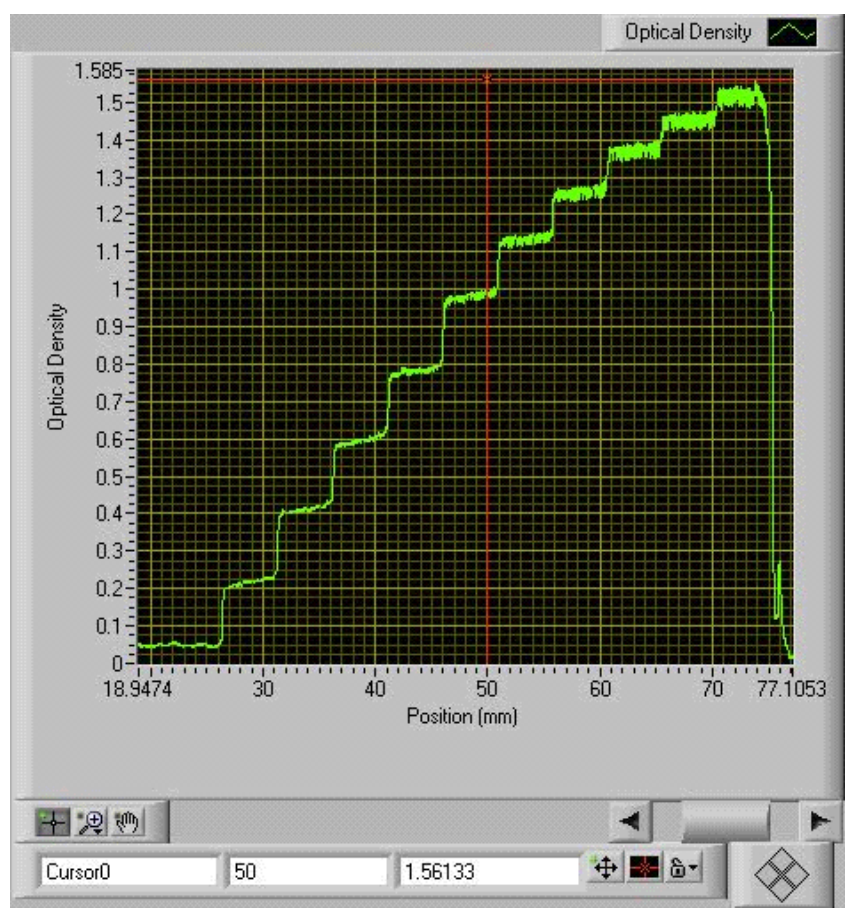

Figure 5. Kodak OD step tablet versus position scan as seen on the PC screen. 
limited by the X-ray source size, which in this case is $100 \mu \mathrm{m}$, larger than the spatial resolution of the instrument $(70 \mu \mathrm{m})$. It may be noted that here the intensity means relative intensities of the lines. If one has data on absolute intensity calibration of the film used, one can get absolute intensities of the spectral lines. This cannot be done by any other instrument like a standard scanner, as these instruments operate in reflection mode and not in transmission mode as in a microdensitometer.

The on-line calibration was done by scanning a Kodak make standard OD tablet. It has an OD range from $0 \cdot 05$ to $1 \cdot 55$. Figure 5 shows the scan of Kodak step tablet with respect to the position as displayed by the software on the PC screen.

In conclusion, we have developed a PC-based control software and data acquisition system in our laboratory for a commercial laser scanning microdensitometer. It can scan an X-ray (or visible $\mathrm{B} \& \mathrm{~W}$ ) film in $1 \mathrm{D}$ or $2 \mathrm{D}$ scan mode with a spatial resolution of $\sim 70$ micron (limited by source) and save the same in digital form. It also provides a user-friendly Graphical User Interface for analysis of the scanned data and also saves the analysed data/image in popular formats like data in MS Excel and images in jpeg. It also has a facility for on-line calibration with standard OD tablets. The control software being in Labview, it can be easily upgraded or modified to incorporate any future requirement. The data acquisition and analysis software is also instrument independent and thus can be easily used in other instruments with minor modifications.

Authors wish to thank V Arora and S R Kumbhare for providing help in making measurements on film and in calibration of the system.

\section{References}

Moghe N V, Mathurkar N, Bhongale G M, Sapre V B 1990 Automation and computerization of microdensitometer. Meas. Sci. Technol. 3: 557-559

Vora H S, Upadhyay J, Naik P A, Navathe C P, Gupta P D 2002 A data acquisition and analysis system for online calibration and measurements of optical density by a scanning microdensitometer. IETE Tech. Rev. 19: 65-69 